\title{
Sensitivity and specificity of SARS-CoV-2 S1 subunit in COVID-19 serology assays
}

\author{
Ying Tian ${ }^{1}$, Chaoyang Lian ${ }^{1}$, Yingying Chen ${ }^{1}$, Dong Wei ${ }^{2}$, Xinxin Zhang ${ }^{2}$, Yun Ling ${ }^{3}$, Ying Wang ${ }^{1}$ and Leng-Siew Yeap ${ }^{1}$
}

\section{Dear Editor,}

Serological assays such as enzyme-linked immunosorbent assays (ELISA) using SARS-CoV-2 Spike (S) proteins are practical methods to determine the extent of COVID19 immunity in a population upon SARS-CoV-2 vaccination ${ }^{1}$. However, the robustness of these assays, which depends on the sensitivity and specificity of $\mathrm{S}$ proteins in detecting anti-SARS-CoV-2 antibodies, is not well characterized. Here, we report that the S1 subunit of the SARS-CoV-2 S protein has superiority over the receptorbinding domain (RBD) and the native state $S$ trimer in terms of sensitivity and specificity, respectively, in measuring anti-SARS-CoV-2 antibodies from COVID-19 convalescent patients. S1 and S trimer are more sensitive than RBD antigen because they are able to capture non-RBD, as well as RBD-binding COVID-19 antibodies. However, the full-length $\mathrm{S}$ trimer, which harbors S2 subunit in addition to S1, cross-reacted with antibodies elicited by circulating coronavirus $(\mathrm{CoV})$, such as $\mathrm{HCoV}$ OC43 and HCoV-HKU1, making it less specific than $\mathrm{S} 1$ in detecting COVID-19 antibodies. Our results show that the $\mathrm{S} 1$ subunit protein of SARS-CoV-2 is both sensitive and specific in distinguishing COVID-19 antibodies, suggesting its potential implication in large-scale serological testing, especially in determining the efficacy of SARS-CoV-2 vaccine.

\footnotetext{
Correspondence: Yun Ling (yun.ling@shphc.org.cn) or

Ying Wang (ywangssmu@shsmu.edu.cn) or Leng-

Siew Yeap (yeaplengsiew@shsmu.edu.cn)

'Shanghai Institute of Immunology, State Key Laboratory of Oncogenes and Related Genes, Department of Immunology and Microbiology, Shanghai Jiao Tong University School of Medicine, 280 South Chongqing Road, Shanghai 200025, China

${ }^{2}$ Department of Infectious Diseases, Research Laboratory of Clinical Virology, Ruijin Hospital, Shanghai Jiao Tong University School of Medicine, Shanghai 200025, China

Full list of author information is available at the end of the article

These authors contributed equally: Ying Tian, Chaoyang Lian, Yingying Chen
}

$\mathrm{CoV}$ infection has been known to occur in humans. Some human CoV strains that are widely circulating, such as $\mathrm{HCoV}-\mathrm{HKU} 1, \mathrm{HCoV}-\mathrm{OC} 43, \mathrm{HCoV}-\mathrm{NL} 63$, and $\mathrm{HCoV}-$ $229 \mathrm{E}$ caused mild respiratory disease, while other endemic strains, such as SARS-CoV and MERS-CoV caused a more severe disease ${ }^{2}$. The recent outbreak of SARS-CoV2, a virus from the Betacoronavirus family that has high homology to SARS-CoV, caused an unprecedented CoVrelated pandemic, and posed major threat to global health and stability ${ }^{3,4}$. SARS-CoV-2 infection, like other viral infections, triggers adaptive immune response, including generation of neutralizing antibodies ${ }^{5}$. In response to the pandemic, there is a pressing need for extensive serological testing to estimate the levels of COVID-19 antibodies in high-risk communities and to evaluate the effectiveness of SARS-CoV-2 vaccine in generating neutralizing antibodies. Hence, identification of a robust antigen and serological method is important to facilitate large-scale screening of COVID-19 antibodies. The SARS$\mathrm{CoV}-2 \mathrm{~S}$ protein is a good candidate for antigen as many COVID-19 antibodies have been found to target the S protein $^{6-10}$. In this regard, neutralizing antibodies against SARS-CoV-2 or the closely related SARS-CoV mainly target the RBD on the $S 1$ subunit of the $S$ protein (Fig. 1a), a region that engages the receptor, ACE2, on the host cell $^{11}$. The full-length $\mathrm{S}$ protein, which consists of the S1 and S2 subunits, normally exists in a trimeric state with one of the three RBDs being in an accessible conformation $^{12,13}$. Our aim is to determine which components or forms of the $\mathrm{S}$ protein is more sensitive and specific in ELISA assays to detect and differentiate anti-SARS-CoV2-specific antibodies from antibodies elicited by the widely circulating CoVs.

We performed ELISA on plasma of 35 COVID-19 convalescent patients and 18 healthy controls, using SARS-CoV-2 RBD, S1, or S trimer antigens (Fig. 1b). As controls for normalization of OD measurements in

\section{(c) The Author(s) 2020}

(c) (i) Open Access This article is licensed under a Creative Commons Attribution 4.0 International License, which permits use, sharing, adaptation, distribution and reproduction in any medium or format, as long as you give appropriate credit to the original author(s) and the source, provide a link to the Creative Commons license, and indicate if changes were made. The images or other third party material in this article are included in the article's Creative Commons license, unless indicated otherwise in a credit line to the material. If material is not included in the article's Creative Commons license and your intended use is not permitted by statutory regulation or exceeds the permitted use, you will need to obtain permission directly from the copyright holder. To view a copy of this license, visit http://creativecommons.org/licenses/by/4.0/. 

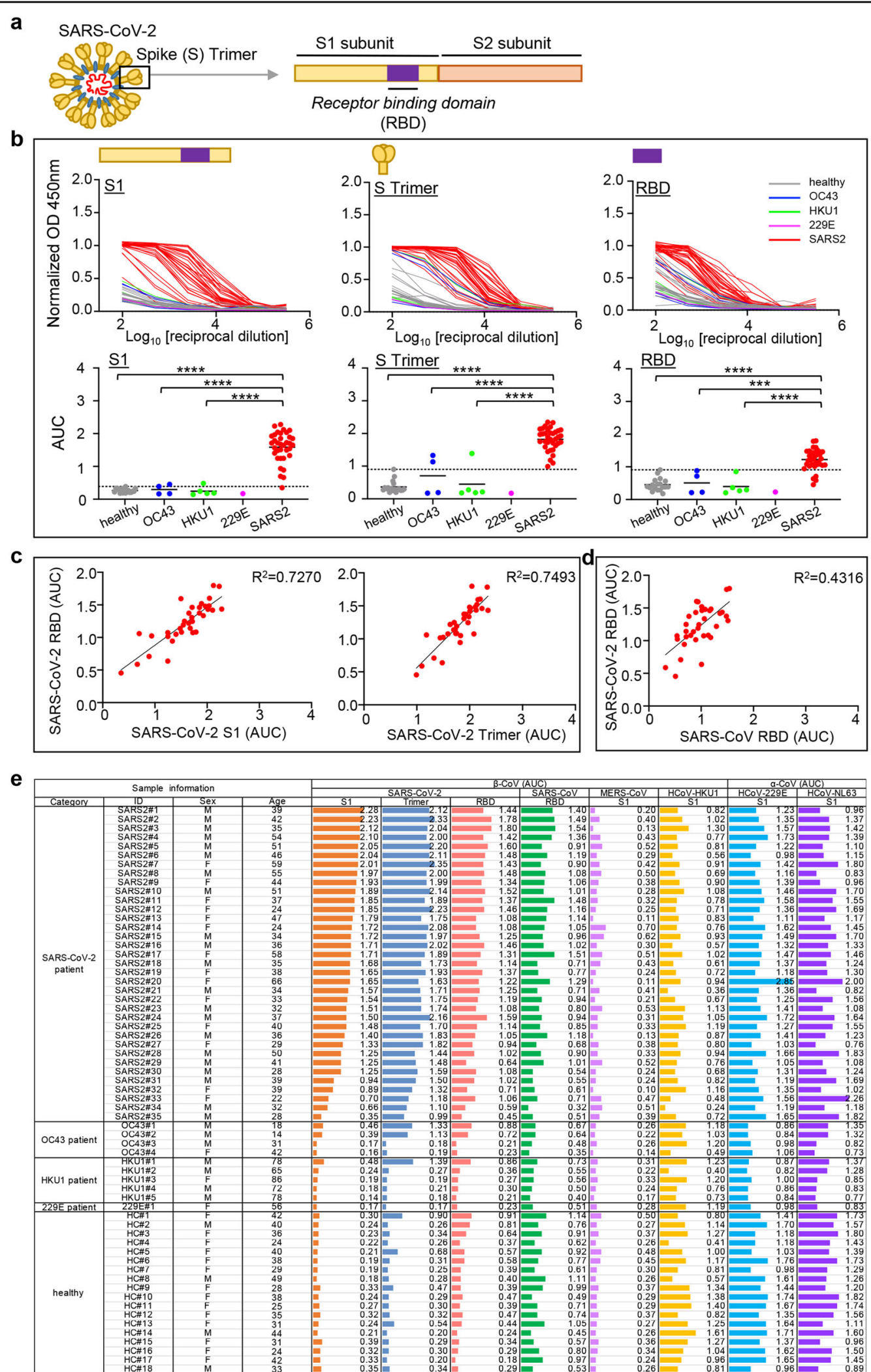

Fig. 1 (See legend on next page.) 
(see figure on previous page)

Fig. 1 ELISA assays to detect anti-SARS-CoV-2 antibodies in human coronaviruses infected individuals. a Schematic showing SARS-CoV-2 S trimer, S1 and RBD. b ELISA measurement of plasma reactivity to RBD, S1, and S trimer. Y-axis is OD $450 \mathrm{~nm}$ normalized to the mean absorbance of CR3022 in each plate. $X$-axis is log reciprocal plasma dilution (upper row). Normalized area under the curve (AUC) of 18 controls (gray) and 45 patients (35 patients infected with SARS-CoV-2, red; 4 patients infected with HCoV-OC43, blue; 5 patients infected with HCoV-HKU1, green; and 1 patient infected with HCoV-229E, magenta) for lgG binding to SARS-CoV-2 S1, S trimer, and RBD (lower row). Data were expressed as mean. ${ }^{*} P<0.05$, ${ }^{* *} P<$ $0.01,{ }^{* * *} P<0.001,{ }^{* * *} P<0.0001$. c Correlation between AUC of anti-SARS-CoV-2 RBD and AUC of anti-SARS-CoV-2 S1 (left) or anti-SARS-CoV-2 S trimer (right). $\mathbf{d}$ Correlation between AUC of anti-SARS-CoV-2 RBD and AUC of anti-SARS-CoV RBD. e Demographic details and normalized AUC of all samples for lgG binding to $\beta$-CoV (SARS-CoV-2 S1, S trimer, RBD, SARS-CoV RBD, MERS-CoV S1, and HCoV-HKU1 S1) and a-CoV (HCoV-229E S1 and HCOV-NL63 S1) presented in bar chart.

different plates, we included purified CR3022, an antibody specific to SARS-CoV that also cross-binds to SARS-CoV$2^{14}$ in every plate (Supplementary Fig. S1a). We then calculated the area under the curve (AUC) of the normalized OD using two different methods, first, where the $X$-axis is the log of reciprocal dilution (Fig. 1b) and second, where the $X$-axis is reciprocal dilution (Supplementary Fig. S1b, c). The former method is better than the latter in discerning COVID-19 antibodies from convalescent patient and is used in subsequent analysis. In the former method, S1 and S trimer are both sensitive antigens for detecting COVID-19 antibodies, as all 35 COVID-19 patients had AUC levels above or close to the borderline of the healthy control threshold, which is determined by the highest AUC in the healthy control (Fig. 1b, bottom panel). In the RBD group, the AUC levels of four COVID-19 patients fall below the healthy control threshold. Since S1 and trimer contain other epitopes besides RBD, the sensitivity of these antigens over RBD may be due to their ability to capture non-RBD-binding antibodies. Nevertheless, S1 and S trimer mainly detect COVID-19 antibodies targeting the RBD, as we observed positive correlation between AUC levels measured by RBD and S1 or S trimer (Pearson correlation $=0.7270$ and 0.7493), respectively (Fig. 1c). Therefore, by using an appropriate method of AUC calculation, we show that S1 and $\mathrm{S}$ trimer are better antigens than the RBD in terms of sensitivity against COVID-19 antibodies.

To determine whether the antigens that were tested cross-bind with antibodies elicited by other widely circulating CoVs, we also included samples from patients that were infected with either HCoV-OC43 $(n=4)$, HCoV-HKU1 $(n=5)$, or HCoV-229E $(n=1)$, which were collected prior to SARS-CoV-2 outbreak. For HCoVHKU1 and HCoV-229E, we also performed ELISA with their corresponding S1 antigens. We observed varying degrees of binding activities to their own antigens, similar to the variation that was observed for binding activities of SARS-CoV-2-infected samples to SARS-CoV-2 antigens. Our analysis shows that $\mathrm{S}$ trimer may cross-bind at low levels with antibodies elicited by other circulating $\mathrm{CoV}$ strains, such as those from the Betacoronavirus family HCoV-OC43 and HCoV-HKU1 (Fig. 1b). This is perhaps due to higher homology of the S2 portion of the trimer between SARS-CoV-2 and $\mathrm{HCoV}-\mathrm{OC} 43$ and $\mathrm{HCoV}$ HKU1 compared to the S1 portion alone (Supplementary Fig. S1d). Thus, with respect to the specificity of the antigen toward COVID-19 antibodies, the S1 antigen surpasses the $\mathrm{S}$ trimer. Taken together, the $\mathrm{S} 1$ antigen performed better than the RBD, and $S$ trimer in both sensitivity and specificity towards COVID-19 antibodies.

We also tested whether antibodies from COVID-19 patients cross-reacted with antigens from other CoV. We performed ELISA using plasma from the same patients with SARS-CoV RBD and S1 subunits of MERS-CoV, HCoV-HKU1, HCoV-229E, and HCoV-NL63. The latter two are circulating $\mathrm{CoV}$ from the Alphacoronavirus family. Our results show that SARS-CoV RBD which has $73.8-74.9 \%$ amino acid identity with SARS-CoV-2 RBD ${ }^{4}$ cross-binds with antibodies from COVID-19 patients, as there is a positive correlation (Pearson correlation = 0.4316) between AUC levels measured by SARS-CoV-2 RBD and SARS-CoV RBD (Fig. 1d). This is expected considering the similarity of the RBD sequence between these two strains and that antibodies elicited by SARS$\mathrm{CoV}$-infected patients can cross-neutralize SARS-CoV- $2^{8}$. Antigens from the circulating human $\mathrm{CoVs}$, including HCoV-HKU1, HCoV-229E, and HCoV-NL63 seemed to bind antibodies from COVID-19 patients, as well as healthy controls in varying degree, suggesting prior widespread infection of these CoVs in the general population (Fig. 1e and Supplementary Fig. S2a, b). S1 antigen from MERS-CoV, a virus from the Betacorovirus that caused severe disease and originated from the Middle East generally does not cross-react with antibodies in our cohort of COVID-19 patients and controls (Fig. 1e and Supplementary Fig. S2a). We have not observed positive correlation between AUC levels measured by S1 of SARS$\mathrm{CoV}-2$ and $\mathrm{S} 1$ antigen of other tested CoV strains, suggesting that the latter antigens are probably not specific to COVID-19-specific antibodies (Supplementary Fig. S3). However, it is possible that some COVID-19-specific antibodies in certain convalescent patients may crossreact with the circulating mild $\mathrm{CoV}$ strains. Further experiments would be required to isolate such crossreactive antibodies. 
Our results show that SARS-CoV-2 S1 is a robust antigen in serological assays to detect SARS-CoV-2specific antibodies. S1, being more sensitive than RBD, and more specific than $\mathrm{S}$ trimer, is the optimal antigen among the three SARS-CoV-2 S antigens tested. Recently, SARS-CoV-2-neutralizing antibodies targeting the $\mathrm{N}$ terminal region (NTD) of S1 were isolated using the $\mathrm{S}$ trimer ${ }^{15}$, suggesting that the $\mathrm{S}$ trimer may be better in capturing COVID-19 antibodies. While the native state of $\mathrm{S}$ trimer may be in the prime conformation to capture all SARS-CoV-2-specific antibodies, including those that target regions outside the $\mathrm{RBD}$ and $\mathrm{S} 1^{15}$, it also binds nonspecifically to antibodies elicited by other closely related circulating CoV strains. Thus, when considering both sensitivity and specificity criteria of the antigens in serological assays, S1 antigen is preferable over S trimer. Recent studies have shown that a two-step procedure using both SARS-CoV-2 RBD and S trimer antigens in immunoassays demonstrated high sensitivity and specificity for COVID-19 antibodies ${ }^{16,17}$. In this regard, our results show that $\mathrm{S} 1$ antigen alone can achieve high sensitivity and specificity for detecting COVID-19 antibodies, thereby increasing efficiency and reducing cost of the immunoassays. The ease of purification of $\mathrm{S} 1$ protein over the full-length $\mathrm{S}$ protein or the trimeric form also makes it favorable for large-scale serological testing. While the S1 antigen of SARS-CoV-2 is able to distinguish antibodies targeting SARS-CoV-2 from the widely circulating CoV, it may not be able to distinguish antibodies targeting SARS$\mathrm{CoV}$, considering the high similarity in the amino acid identity between these two strains (Supplementary Fig. S1d). In this case, further experiments using pseudovirus neutralization may be required when determining COVID-19 antibodies from a certain population that may have been previously exposed to SARS-CoV. Another caveat in this study is the relatively small sample size studied. Finally, we propose that S1 antigen is the optimal reagent in facilitating large-scale serological assays in the evaluation of various SARS-CoV-2 vaccines that are currently in development.

\section{Acknowledgements}

This work was supported by funds from National Natural Science Foundation of China (31722020, 81671634, and 81861138014), Shanghai Jiao Tong University COVID-19 grant and The SII Challenge Fund for COVID-19 Research to L.-S.Y., Science and Technology Commission of Shanghai Municipality (20JC1410200) and Shanghai Academic Research Leader Project (2018XD1403300) to Y.W., and China Postdoctoral Science Foundation (2020T130078ZX) and National Natural Science Foundation of China (32000656) to Y.T.

\section{Author details}

${ }^{1}$ Shanghai Institute of Immunology, State Key Laboratory of Oncogenes and Related Genes, Department of Immunology and Microbiology, Shanghai Jiao
Tong University School of Medicine, 280 South Chongqing Road, Shanghai 200025, China. ${ }^{2}$ Department of Infectious Diseases, Research Laboratory of Clinical Virology, Ruijin Hospital, Shanghai Jiao Tong University School of Medicine, Shanghai 200025, China. ${ }^{3}$ Department of Infectious Disease, Shanghai Public Health Clinical Center, Shanghai 201052, China

\section{Author contributions}

Y.T., C.L., Y.W., and L.-S.Y. designed the experiment and wrote the manuscript. Y.Y.C. and and D.W. collected the clinical samples. Y.L. and X.X.Z. selected the patients and collected the clinical data.

\section{Conflict of interest}

The authors declare that they have no conflict of interest.

\section{Publisher's note}

Springer Nature remains neutral with regard to jurisdictional claims in published maps and institutional affiliations.

Supplementary Information accompanies the paper at (https://doi.org/ 10.1038/s41421-020-00224-3).

Received: 18 August 2020 Accepted: 1 October 2020

Published online: 27 October 2020

\section{References}

1. Krammer, F. \& Simon, V. Serology assays to manage COVID-19. Science $\mathbf{3 6 8}$, 1060-1061 (2020).

2. Song, Z. et al. From SARS to MERS, thrusting coronaviruses into the spotlight. Viruses 11, 59 (2019).

3. Zhou, P. et al. A pneumonia outbreak associated with a new coronavirus of probable bat origin. Nature $\mathbf{5 7 9}, \mathbf{2 7 0 - 2 7 3 ~ ( 2 0 2 0 ) . ~}$

4. Wu, F. et al. A new coronavirus associated with human respiratory disease in China. Nature 579, 265-269 (2020).

5. $\mathrm{Ni}$, L. et al. Detection of SARS-CoV-2-specific humoral and cellular immunity in COVID-19 convalescent individuals. Immunity 52, 971-977 (2020).

6. Ju, B. et al. Human neutralizing antibodies elicited by SARS-CoV-2 infection. Nature 584, 115-119 (2020).

7. Cao, Y. et al. Potent neutralizing antibodies against SARS-CoV-2 identified by high-throughput single-cell sequencing of convalescent patients' B cells. Cell 182, 73-84 (2020).

8. Pinto, D. et al. Cross-neutralization of SARS-CoV-2 by a human monoclonal SARS-CoV antibody. Nature 583, 290-295 (2020).

9. Wu, Y. et al. A noncompeting pair of human neutralizing antibodies block COVID-19 virus binding to its receptor ACE2. Science 368, 1274-1278 (2020).

10. Hansen, J. et al. Studies in humanized mice and convalescent humans yield a SARS-CoV-2 antibody cocktail. Science 369, 1010-1014 (2020).

11. Yuan, M. et al. A highly conserved cryptic epitope in the receptor binding domains of SARS-CoV-2 and SARS-CoV. Science 368, 630-633 (2020).

12. Yan, R. et al. Structural basis for the recognition of SARS-CoV-2 by full-length human ACE2. Science 367, 1444-1448 (2020).

13. Wrapp, D. et al. Cryo-EM structure of the 2019-nCoV spike in the prefusion conformation. Science 367, 1260-1263 (2020).

14. Tian, X. et al. Potent binding of 2019 novel coronavirus spike protein by a SARS coronavirus-specific human monoclonal antibody. Emerg. Microbes Infect. 9, 382-385 (2020).

15. Liu, L. et al. Potent neutralizing antibodies against multiple epitopes on SARSCoV-2 spike. Nature 584, 450-456 (2020).

16. Klumpp-Thomas, C., Kalish, H. \& Drew, M. Standardization of enzyme-linked immunosorbent assays for serosurveys of the SARS-CoV-2 pandemic using clinical and at-home blood sampling. Preprint at https://doi.org/10.1101/ 2020.05.21.20109280 (2020).

17. Amanat, F. et al. A serological assay to detect SARS-CoV-2 seroconversion in humans. Nat. Med. 26, 1033-1036 (2020). 